\title{
Importancia de la vigilancia del desarrollo psicomotor por el pediatra de Atención Primaria: revisión del tema y experiencia de seguimiento en una consulta en Navarra
}

\author{
MJ. Álvarez Gómez $z^{\mathrm{a}}$, J. Soria Aznar ${ }^{\mathrm{b}}$, J. Galbe Sánchez-Ventura ${ }^{\mathrm{c}}$ \\ Grupo de Pediatría del Desarrollo de la Asociación Española \\ de Pediatría de Atención Primaria (AEPap) ${ }^{d}$ \\ ${ }^{a}$ Pediatra. CS Mendillorri. Servicio Navarro Salud-Osasunbidea. Navarra. \\ ${ }^{b}$ Pediatra. CS Chantrea. Servicio Navarro Salud-Osasunbidea. Navarra. \\ 'Pediatra. CS Torrero-La Paz. Zaragoza.
}

Rev Pediatr Aten Primaria. 2009; I :65-87

María José Álvarez Gómez, malvareg@cfnavarra.es

\section{Resumen}

La detección precoz de los niños con retrasos/trastornos del desarrollo o con factores de riesgo para dicho desarrollo, es importante en nuestra práctica como pediatras de Atención Primaria. La vigilancia del desarrollo en las visitas de salud programadas, constituye una oportunidad ideal para la identificación precoz de los niños con problemas de desarrollo de cara a realizar una derivación a tiempo para un diagnóstico preciso y para recibir Atención Temprana.

Presentamos un estudio retrospectivo de 472 niños nacidos entre 1996 y 2002, seguidos durante 6 años, en una consulta de Pediatría de Atención Primaria en Navarra.

El 8,68\% de los niños de 0-6 años presentaron retrasos/trastornos del desarrollo o tenían factores de riesgo para el mismo. A lo largo del seguimiento el 51,21\% de ellos han padecido problemas del desarrollo. Añadiendo en el seguimiento a 6 años a la población infantil restante, que no parecía necesitar una vigilancia especial, el 11,2\% de niños de 0-6 años presentaron problemas del desarrollo o trastornos de aprendizaje.

Palabras clave: Vigilancia del desarrollo, Monitorización del desarrollo, Cribado del desarrollo, Retraso del desarrollo, Trastornos del desarrollo, Atención Temprana.

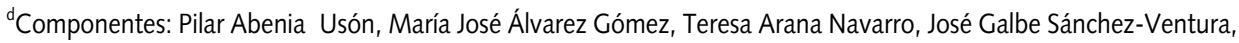
Beatriz Garnica Martínez, Juan José Morell Bernabé, Ana Pérez Salas, Antonio Pons Tubío, Rosa Rodríguez Posadas, Juan Carlos Sanguino Fabre, Jesús Soria Aznar.
}

Los autores declaran no presentar conflictos de intereses en relación con la preparación y publicación de este artículo. 


\section{Abstract}

The early identification of children with developmental delays/disorders or with risk factors for development outcomes is important in our practice as primary care pediatricians. Developmental surveillance in well-child visits provides an ideal opportunity for early identification of children with developmental difficulties in order to make a timely referral for accurate diagnosis and early intervention programs.

This is a retrospective study of 472 children 0-6 years born between 1996 and 2002, followed up in a primary care practice in Navarra during 6 years.

Eight and sixty-eight percent of children 0-6 years presented developmental delays / disorders or risk factors for development outcomes. Along the follow up, $51.21 \%$ of them have suffered from some development difficulties. Adding the remainder normal population who didn't seem to need special surveillance to the six years follow up, $11.2 \%$ of children 0-6 years presented with some developmental or learning disabilities.

Key words: Developmental surveillance, Developmental screening, Developmental Delay, Developmental Disorders, Early Intervention.

\section{Introducción}

La detección precoz de los problemas del desarrollo es un procedimiento diseñado para identificar aquellos niños que deben recibir una valoración más exhaustiva -un diagnóstico- de un posible retraso o trastorno del desarrollo.

En Estados Unidos se utiliza más acertadamente el término "vigilancia del desarrollo" (surveillance) para describir esta actuación tan propia de la Pediatría; y que Dworkin describe como "un proceso continuo, flexible, a través del cual profesionales con conocimientos y entrenamiento realizan observaciones de los niños durante las revisiones de salud" y cuyos componentes serían entre otros: "saber inquirir y atender a las preocupaciones de los padres, obtener la historia del desarrollo, hacer observaciones precisas del niño y compartir opiniones y preocupaciones con otros profesionales pertinentes"1.

Sin embargo, a pesar de que hace más de tres décadas que profesionales como Illingworth y Haggerty señalaron una nueva morbilidad en la práctica pediátrica en forma de problemas del desarrollo, de la conducta o del ámbito psicosocial, así como la necesidad de que el pediatra adquiriese nuevas habilidades para su diagnóstico, todavía resulta difícil de precisar en la actualidad cuál es la mejor manera de identificar a los niños con dichos problemas del desarrollo $0^{1,2}$.

\section{Terminología: "retraso" o}

"trastorno" del desarrollo

Ambos términos implican que un niño no alcanza un desarrollo similar al de la mayoría de sus pares de la misma edad 
cronológica. Sin embargo, son términos que implican entidades diferentes:

- Retraso y retardo del desarrollo son sinónimos y tienen que ver con una demora o lentitud en la secuencia normal de adquisición de los hitos del desarrollo.

- Trastorno y alteración del desarrollo son también sinónimos pero se refieren, en cambio, a una perturbación, cambio en la esencia o patrón anormal del desarrollo.

En el retraso del desarrollo no hay nada intrínsecamente anormal, ya que los hitos madurativos se cumplen en la secuencia esperada solo que de forma más lenta, de modo que se comporta como un niño menor respecto a su edad cronológica. Sin embargo, en el caso del trastorno del desarrollo el patrón no se produce en la secuencia esperada, siendo intrínsecamente anormal para cualquier edad; el paradigma de los trastornos del desarrollo es el autismo.

Por otra parte, el retraso puede afectar a una sola área del desarrollo -por ejemplo la motricidad o el lenguaje-. Pero cuando el retraso afecta a dos o más áreas del desarrollo hablamos de retraso global del desarrollo4.

En nuestro país se emplea el término retraso psicomotor como sinónimo de retraso del desarrollo ${ }^{5}$, mientras que en Latinoamérica se denomina retraso madurativo ${ }^{3}$.

Lo más importante, quizás, es que el término retraso del desarrollo es un diagnóstico temporal, hasta que puede establecerse un diagnóstico definitivo a través de pruebas formales, ya sea de normalidad si el retraso se resuelve con el tiempo, o bien de deficiencia mental u otra patología ${ }^{6}$. Esto nos obliga a ser cautos, especialmente en los casos de retraso leves, y también a no utilizar dicho término más allá de los 3 o como mucho de los 5 años de edad del niño cuando ya se pueden realizar test que miden la capacidad intelectual ${ }^{5,7}$.

\section{Importancia del problema: incidencia y prevalencia de los problemas del desarrollo}

En Estados Unidos se estima que entre un 1 y un $3 \%$ de los niños menores de 5 años presentan retraso del desarro$110^{4}$; y que el $5-10 \%$ de la población pediátrica sufre alguna "discapacidad del desarrollo" ${ }^{4,8}$. Si añadimos los problemas de comportamiento, resulta que el $12-16 \%$ de los niños padece problemas de desarrollo o de conducta ${ }^{9}$ y la prevalencia aumenta hasta el $16,8 \%$ si consideramos los 15 primeros años de edad $^{10}$. Es decir, en Norteamérica se 
diagnostican problemas del desarrollo y/o de comportamiento en 1 de cada 6 niños.

En España, la encuesta sobre discapacidades, deficiencias y estado de salud realizada por el Instituto Nacional de Estadística en 1999 concluye que el 2,24\% de niños de 0 a 6 años de edad tiene una limitación; el 2,5\% de niños de esta edad tiene un trastorno del desarrollo que no va a derivar forzosamente en discapacidad y otro $2,5 \%$ de niños presenta riesgo biológico o social de padecer un trastorno ${ }^{11}$. Se prescinde en este trabajo del estudio pormenorizado de las discapacidades de los niños, alegando la dificultad que tiene su detección a estas edades. De cualquier modo estas cifras nos sitúan ante más de un $7 \%$ de niños que preci- san seguimiento $y / 0$ tratamiento en nuestras consultas. Puede, por tanto, deducirse que estamos ante un problema de salud infantil verdaderamente prevalente.

\section{Material y métodos}

Presentamos nuestra experiencia en una consulta de Pediatría de Atención Primaria -Centro de Salud de Mendillorri- en la ciudad de Pamplona. La muestra está constituida por 472 niños de 06 años nacidos entre 1996 y 2002 y seguidos hasta la actualidad en dicha consulta.

Para la vigilancia del desarrollo se utilizó la anamnesis a los padres y la observación directa del niño en consulta, así como la Escala de Haizea-Llevant ${ }^{12}$

Figura 1. Población infantil 0-6 años $(n=472)$ con retraso/trastorno del desarrollo o en situación de riesgo.

41

431 $91,32 \%$ 
como instrumento de cribado para las edades 0-5 años. En los niños con sospecha de problemas de desarrollo se utilizó como instrumento de diagnóstico para las edades 0-30 meses el test de Bayley ${ }^{13}$. Para el seguimiento en edades superiores se utilizó la anamnesis a los padres y la observación directa del niño en consulta y psicometría convencional de los niños con sospecha de patología, realizada por los orientadores escolares del centro académico de los casos.

\section{Resultados}

De esa cohorte, 41 niños presentaron retrasos y/o problemas del desarrollo o bien se encontraban en situación de riesgo -biológico y/o social- para el desarrollo (tabla I). En estos últimos se realizó un seguimiento más estrecho por parte del pediatra, y algunos casos requirieron algún tipo de tratamiento específico. En conjunto, el número de casos detectados en nuestra consulta supone el $8,68 \%$ de la población general de 0-6 años (figura 1).

Respecto al tratamiento, 22 niños (54\%) precisaron vigilancia por el pediatra de Atención Primaria (AP): en 8 niños (36\%) únicamente fue necesario hacer seguimiento del desarrollo sin ninguna intervención y en los 14 casos restantes (64\%) el pediatra dio a los padres pautas sobre la estimulación del niño siguiendo el programa de evaluación y ejercicios para bebés y niños pequeños con necesidades especiales ("Currículo Carolina") 14 . El otro $46 \%$ de niños precisaron derivación para tratamiento más específico, bien al Centro Base del Instituto Navarro de Bienestar Social, o bien a Rehabilitación, Psiquiatría Infantojuvenil, escue-

Tabla I. Niños con problemas de desarrollo o con factores de riesgo para el desarrollo

Características de la muestra $(n=41)$

Sexo

- Niños

- Niñas

Factores de riesgo

- Sin factores de riesgo

- Con factores de riesgo

-1 factor de riesgo

- 5 factores de riesgo

- Factores de riesgo biológico

- Factores de riesgo social
$\%$ de la muestra

59

41

27

73

63

3

76 (24\% prematuridad/bajo peso)

24 
las infantiles o una combinación de estos recursos.

Respecto a la evolución, 7 niños han cambiado de domicilio y se ha conse- guido contactar nuevamente con 6 de ellos; esto supone que hay un $2,4 \%$ de casos perdidos en la muestra.

De los niños con retrasos/problemas

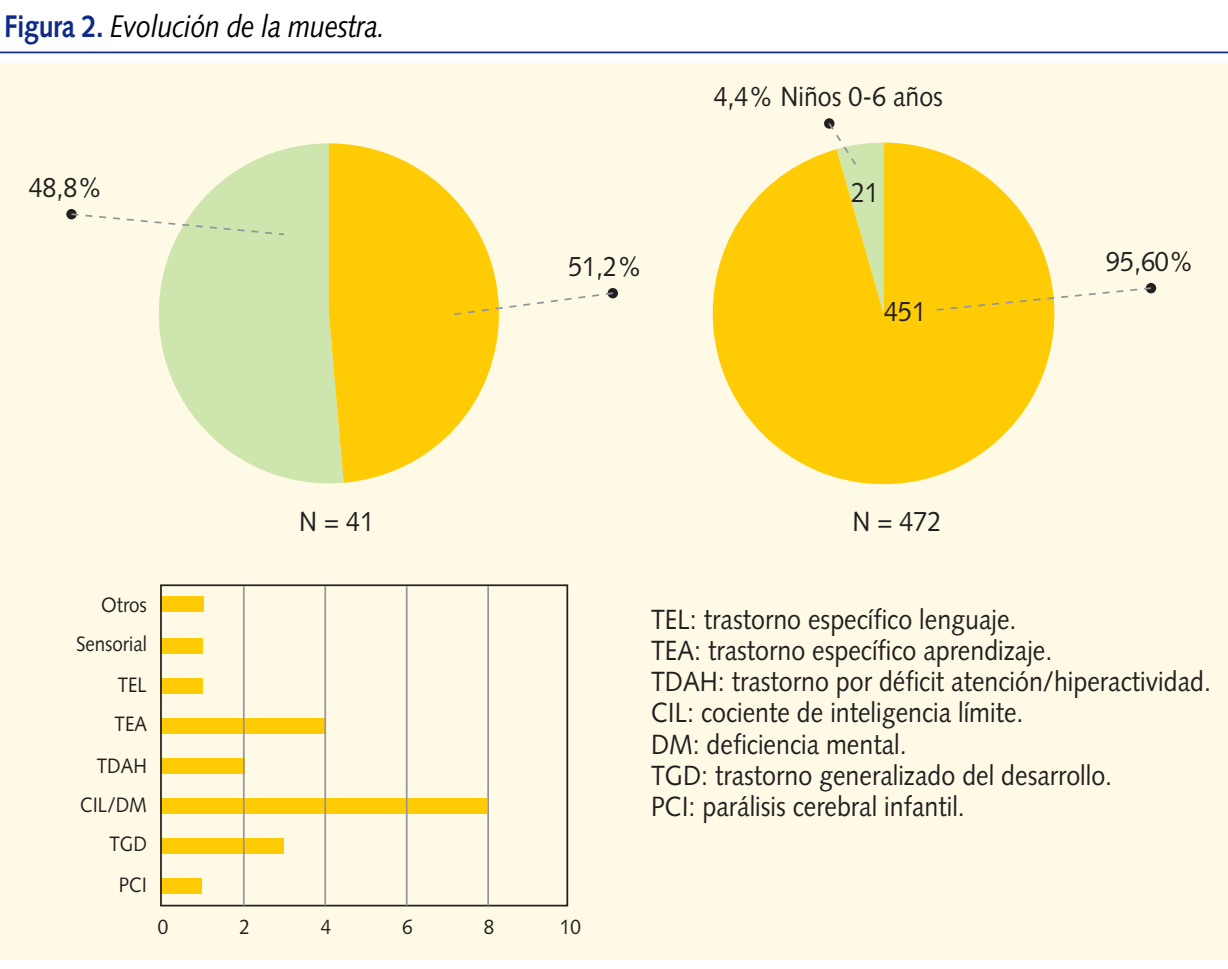

Tabla II. Evolución de los niños con problemas de desarrollo o con factores de riesgo para el desarrollo

Evolución de la muestra $(n=21)$

- Parálisis cerebral infantil

- Trastorno generalizado del desarrollo

- Cl límite/deficiencia mental

- TDAH

- Trastorno específico aprendizaje

- Trastorno específico lenguaje

- Patología neurosensorial

- En evaluación

\section{N. ${ }^{\circ}$ niños}

1 (diplejia espástica )

3

8

2

4

1 (disfasia mixta)

1 (hipoacusia neurosensorial)

1 (¿síndrome de Asperger?)

Cl: cociente de inteligencia; TDAH: trastorno por déficit de atención e hiperactividad. 
del desarrollo o en situación de riesgo para dicho desarrollo, 21 casos $(51,21 \%$ de la muestra) han evolucionado con algún tipo de patología (tabla II). Esto representa el $4,4 \%$ de la población general de 0-6 años (figura 2).

Hay que resaltar que evolucionaron hacia algún tipo de patología tanto los niños con factores de riesgo ( $54 \%$ de casos) como los niños que carecían de factores de riesgo ( $50 \%$ de estos). También que de los niños que no accedieron a tratamiento de Atención Temprana el 36,3\% evolucionó hacia algún tipo de patología.
Es interesante además valorar la evolución del resto de los niños de 0-6 años que no precisaron seguimiento especial por el pediatra de AP. De ellos, 32 niños presentaron también problemas del desarrollo a lo largo de estos 6 años de seguimiento: 13 niños padecen trastorno por déficit de atención con hiperactividad (TDAH); 10 niños, trastorno específico de aprendizaje; 5 niños, con cociente intelectual límite o deficiencia mental ligera; y 4 niños, un trastorno específico de lenguaje. Lo que supone finalmente que a lo largo de estos años de evolución el

Figura 3. Evolución de la población general.

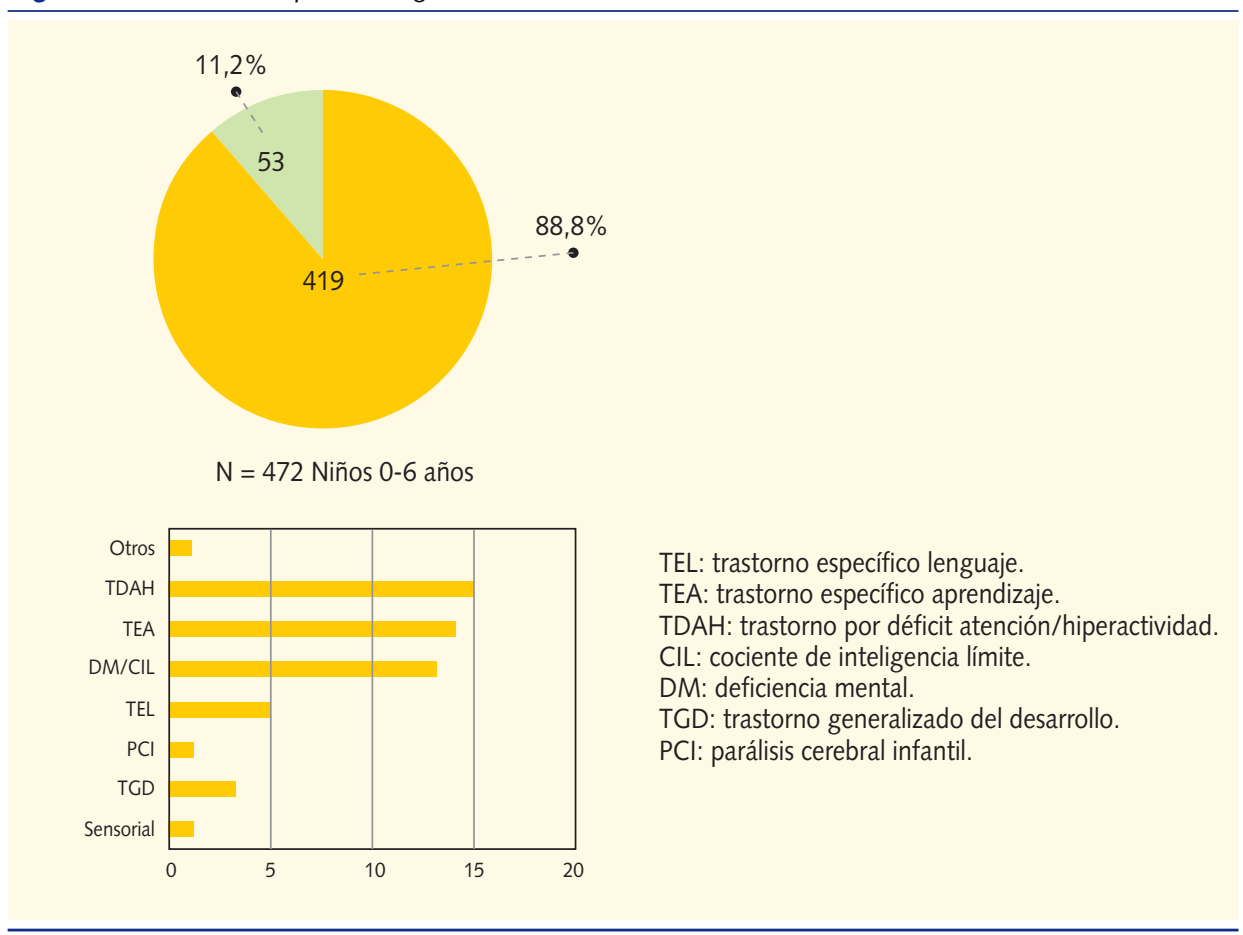


11,2\% de la población de niños de 0-6 años presenta algún tipo de problema del desarrollo (figura 3).

\section{Discusión}

\section{Importancia del diagnóstico precoz} de los problemas del desarrollo

Nuestra muestra confirma la alta prevalencia de problemas del desarrollo que se refieren en estas edades. Pero, ¿es importante el diagnóstico precoz de los mismos? Ciertamente no existe "cura" para todos los casos de alteraciones del desarrollo pero el tratamiento puede optimizar el curso del mismo y mejorar el pronóstico de estos niños y la calidad de vida de sus familias ${ }^{15}$. Podemos actuar a varios niveles:

- Prevención primaria: actuación sobre los niños de riesgo biológico y/o social mediante la disminución de dichos factores de riesgo y el incremento de los factores protectores.

- Prevención secundaria: actuación sobre los niños con retraso del desarrollo mejorando su funcionamiento y disminuyendo el grado de retraso.

- Tratamiento compensador: actuación sobre niños con discapacidades establecidas (síndrome de
Down, parálisis cerebral infantil, etc.), no podemos cambiar la patología pero podemos mejorar la calidad de vida del niño y su familia.

Actualmente, el tratamiento -conjunto de actuaciones- disponible recibe el nombre de Atención Temprana (aunque ya desde finales de los años 70 y con mayor énfasis en la década de los 80 se implantaron en nuestro país numerosos centros dedicados a la detección precoz, diagnóstico y tratamiento de niños con problemas en el desarrollo y que recibieron entonces el nombre de "centros de estimulación precoz").

Se entiende por Atención Temprana el conjunto de intervenciones dirigidas a la población infantil de 0-6 años, a la familia y al entorno, que tienen por objetivo dar respuesta lo más pronto posible a las necesidades transitorias o permanentes que presentan los niños con trastornos en su desarrollo o que tienen riesgo de padecerlos ${ }^{16}$.

La importancia del diagnóstico y de la atención, ambos precoces, estriba en que hay evidencia de los beneficios de la Atención Temprana y, por tanto, posibilidad de mejorar el pronóstico de estos niños ${ }^{16-19}$, si bien es cierto que hay una extraordinaria variabilidad entre los 
distintos programas de intervención evaluados, lo que hace que las conclusiones sobre su eficacia estén todavía en proceso de investigación. En líneas generales los metaanálisis concluyen que la Atención Temprana tiene un efecto positivo, aunque moderado, que es más eficaz cuando los programas son estructurados, intensos e incluyen a la familia; con efectos más manifiestos a corto y medio plazo. En la actualidad hay solo dos programas de buena calidad metodológica con un seguimiento lo suficientemente largo: el Abecedarian Project ${ }^{20,21}$ y el Infant Health Development Project ${ }^{22}$, con un seguimiento a 21 años y 18 años respectivamente, que apuntan que estos efectos, si bien moderados, se mantienen a más largo plazo.

Los factores de riesgo para el desarrollo pueden ser de índole biológico o social y están claramente definidos en el Libro Blanco de la Atención Temprana (tabla III) $^{16}$. Recientemente, la Federación Estatal de Asociaciones de Profesionales de la Atención Temprana (GAT) ha establecido, también, una nueva forma de clasificación: ODAT (organización diagnóstica para la Atención Temprana) ${ }^{23}$.

Según cifras de la OMS entre un 3 y un $5 \%$ de los embarazos se consideran de alto riesgo y un $12 \%$ son de riesgo moderado; un 10-12\% de recién nacidos precisan ingreso en una unidad neonatal y un 3-5\% de recién nacidos son de riesgo neurológico ${ }^{24}$. Pues bien, pese a los adelantos en la asistencia neonatal en estos últimos años, parece que estas cifras se mantienen estables a lo largo del tiempo y, según estudios en nuestro entorno, el 4-4,4\% siguen siendo neonatos de riesgo neurosensorial ${ }^{25}$.

En Estados Unidos el acceso a los servicios de Atención Temprana para los niños con discapacidades, con problemas de desarrollo o en situación de riesgo de padecerlos está legislado ${ }^{26}$; pese a todo ello, solo el 2,3\% de los niños menores de 3 años recibe atención temprana en este país (variaciones entre el 1,28-7,09\% en diferentes estados) cuando el $5,87 \%$ de niños precisa este tipo de atención en preescolar ${ }^{2,27}$ lo que nos indica que los niños que participan de los programas de Atención Temprana representan solo una pequeña proporción de los niños con problemas del desarrollo o lo que es lo mismo: no se identifica un número significativo de niños que podrían beneficiarse de esta intervención.

En España, con la Ley de Integración Social de los Minusválidos (LISMI) de 1982 se hace ya mención a la prevención y pronta actuación ante la aparición de 
Tabla III. Factores de riesgo para el desarrollo

\section{Factores de riesgo biológico}

A. Recién nacido de riesgo neurológico

- Recién nacido con peso $<$ p10 para su edad gestacional o con peso $<1.500$ g o edad gestacional $<32$ semanas*

- Apgar $<3$ al minuto $0<7$ a los 5 minutos

- Recién nacido con ventilación mecánica durante más de 24 horas

- Hiperbilirrubinemia que precise exanguinotransfusión

- Convulsiones neonatales

- Sepsis, meningitis o encefalitis neonatal

- Disfunción neurológica persistente (más de 7 días)

- Daño cerebral evidenciado en pruebas de imagen

- Malformaciones del sistema nervioso central

- Neurometabolopatías

- Cromosomopatías y otros síndromes dismórficos

- Hijo de madre con patología mental y/o infecciones y/o drogas que puedan afectar al feto

- Recién nacido con hermano con patología neurológica no aclarada o con riesgo de recurrencia

- Gemelo, si el hermano presenta riesgo neurológico

- Siempre que el pediatra lo considere oportuno

B. Recién nacido de riesgo sensorial-visual

- Ventilación mecánica prolongada

- Gran prematuridad

- Recién nacido con peso $<1.500 \mathrm{~g}$

- Hidrocefalia

- Infecciones congénitas del sistema nervioso central

- Patología craneal detectada en pruebas de imagen

- Síndrome malformativo con compromiso visual

- Infecciones posnatales del sistema nervioso central

- Asfixia severa

C. Recién nacido de riesgo sensorial-auditivo

- Hiperbilirrubinemia que precisa exanguinotransfusión

- Gran prematuridad

- Recién nacido con peso $<1.500 \mathrm{~g}$

- Infecciones congénitas del sistema nervioso central

- Ingesta de aminoglucósidos durante un periodo prolongado o con niveles plasmáticos elevados durante el embarazo

- Síndromes malformativos con compromiso de la audición

- Antecedentes familiares de hipoacusia

- Infecciones posnatales del sistema nervioso central

- Asfixia grave 
Tabla III. Factores de riesgo para el desarrollo
2. Factores de riesgo social
- Acusada deprivación económica
- Embarazo accidental traumatizante
- Convivencia conflictiva en el núcleo familiar
- Separación traumatizante en el núcleo familiar
- Padres con bajo coeficiente intelectual/entorno no estimulante
- Enfermedades graves/éxitus
- Alcoholismo/drogadicción
- Prostitución
- Delincuencia/encarcelamiento
- Madres adolescentes
- Sospecha de malos tratos
- Niños acogidos en hogares infantiles
- Familias que no cumplimentan los controles de salud repetidamente

${ }^{*}$ Criterio que debería ser modificado, pues en la experiencia hospitalaria niños < 35 semanas y los $<2.000$ g pueden ser también de alto riesgo.

deficiencias, pero no es hasta que comienzan a aparecer las leyes de servicios sociales de las distintas comunidades autónomas cuando hay ya una referencia expresa a estos servicios. Inicialmente se denominaron "estimulación precoz" y se venían dispensando fundamentalmente en los centros base del entonces INSERSO. Actualmente no hay una uniformidad en la atención entre las diversas comunidades -tanto a nivel de prestaciones como de población infantil a atender-. Finalmente, algunos de los propios profesionales de Atención Temprana se han organizado bajo la denominación de Grupos de Atención Temprana (GAT), y tras reunirse en la sede del Real Patronato sobre Discapacidad en Madrid han elaborado el Libro Blanco de la Atención Temprana para intentar crear una regulación que palie tantas diferencias entre las comunidades ${ }^{16}$.

En nuestra comunidad (Navarra) la Atención Temprana a los niños residentes en Pamplona se realiza, para el tramo de edad 0-3 años, en el centro base dependiente del Instituto de Bienestar Social, y para el tramo de edad comprendido entre los 3-6 años se prestan los apoyos educativos necesarios en los colegios dependiendo del Departamento de Educación ${ }^{28}$; los niños de 0 a 6 años en otras zonas básicas de la provincia (Tudela, Estella, zonas de la Barranca, zona norte de Navarra, Tafalla y Sangüesa) reciben Atención Temprana ade- 
más a través de ANFAS (Asociación Navarra en favor de las personas con discapacidad intelectual) mediante acuerdos de colaboración con el Instituto de Bienestar Social ${ }^{29}$.

En nuestro país la campaña de orientación y sensibilización sobre desarrollo infantil y Atención Temprana, fruto de la colaboración entre FEAPS (Confederación Española de Organizaciones a favor de las personas con discapacidad intelectual), la AEP (Asociación Española de Pediatría) y la Obra Social de Caja Madrid estiman que más del $7 \%$ de niños de 0 a 6 años pueden requerir Atención Temprana ${ }^{30}$ y nos recuerda, nuevamente, la gran desigualdad existente entre comunidades.

\section{¿Qué profesional debe realizar el diagnóstico de los problemas del desarrollo?}

Realmente puede ser realizado por cualquier profesional de la salud, la educación o los servicios sociales, pero parece que el pediatra de Atención Primaria (AP) es el profesional más idóneo para hacer el seguimiento del desarrollo infantil ${ }^{9,31,32}$. Su papel es crucial por la posibilidad para hacer un diagnóstico precoz, lo que ha llevado a las sociedades científicas a recomendar que todos los niños tengan un cribado de retraso de desarro- llo periódicamente en el contexto de las visitas de $A P^{4,9,32}$. Esto es particularmente cierto en el modelo pediátrico de AP en nuestro país, donde somos el primer -y a veces único- referente para muchos niños y familias, tenemos una extraordinaria accesibilidad, conocemos el entorno del niño, tenemos la oportunidad de hacer un seguimiento prolongado de los niños hasta la adolescencia y, además, porque la familia demanda y respeta nuestra autoridad en estos temas.

No obstante, esto también supone que la responsabilidad del diagnóstico precoz recae sobre el pediatra, de quien las propias sociedades científicas y los padres esperan que tenga un vasto conocimiento -que sea un experto- en el campo del desarrollo infantil, que tenga las herramientas necesarias para llegar al diagnóstico, que sepa administrarlas e interpretarlas, que tenga conocimiento sobre los recursos disponibles en su comunidad para tratar estos problemas y que se pueda coordinar con especialistas de otras disciplinas, educación, servicios sociales, atención hospitalaria, etc., que atienden a los niños y a sus familias.

Nada más lejos de ser sencillo. Uno de los grandes retos al evaluar el desarrollo infantil -especialmente en los primeros años- es definir qué constituye la "anormalidad". Un referente de la Neu- 
ropediatría como Aicardi nos recuerda que como pediatras hemos sido formados a partir de casos patológicos, sin haber podido ver qué evolución tuvieron esos niños antes de llegar al diagnóstico, y cuando trabajamos como pediatras de AP tenemos que dilucidar si lo que vemos es preludio de un trastorno del desarrollo o se trata simplemente de una variante benigna del mismo ${ }^{33}$.

La extraordinaria variabilidad interindividual es, además, la regla en el desarrollo infantil. De hecho, cuanto más complejo es el hito, más amplio es el rango de edad en que puede ser adquirido; así, todos sabemos que los niños adquieren, por ejemplo, la sedestación en un estrecho margen de tiempo, mientras que las primeras frases pueden aparecer a cualquier edad entre los 18 meses y los 3 años. Estos márgenes nos obligan a ser prudentes en nuestros diagnósticos; por poner un ejemplo se establece que la marcha autónoma se adquiere entre los 10 y los 16 meses, pero un niño que la adquiera a los 10 meses puede padecer quizá un trastorno por déficit de atención con hiperactividad y otro que inicia la deambulación tan tarde como a los 20 meses puede ser un niño normal e incluso con alta inteligencia pero con historia familiar de marcha autónoma tardía ${ }^{33}$.
Además, la misma terminología "retraso psicomotor" nos indica hasta que punto cargamos la tinta en lo "motor" siendo este aspecto peor predictor del desarrollo que aquellas otras capacidades que están cargadas con más intencionalidad y con más significación cognitiva o relacional que el tono o los hitos puramente motrices ${ }^{33,34}$ y que estamos menos acostumbrados a valorar los pediatras.

Con todas estas premisas, sin embargo, en Estados Unidos se constata que, antes de entrar en la escuela infantil se identifican menos del $30 \%$ de niños con trastornos del desarrollo ${ }^{8,35,36}$. Es importante conocer las barreras que existen para la detección y/o derivación de los problemas del desarrollo: en las encuestas realizadas el $82 \%$ de los pediatras norteamericanos aduce que el principal obstáculo para el diagnóstico de los problemas del desarrollo es la falta de tiempo en las consultas, el $81 \%$ demanda más conocimientos sobre el proceso de Atención Temprana y los recursos disponibles en su comunidad y el $48 \%$ refiere falta de personal para administrar el test de cribado; derivan tan solo un $39 \%$ de los niños con problemas del desarrollo siendo otra de las barreras a la derivación la falta de información desde los servicios de Atención Tempra- 
na sobre los progresos del niño en el $36 \%$ de pediatras encuestados al respecto $^{37}$; derivan más los profesionales del sexo femenino y también los pediatras más que los médicos de familia ${ }^{38}$.

En resumen, los principales motivos que limitan la detección precoz y/o el tratamiento de los problemas del desarrollo son: falta de tiempo, falta de conocimientos, falta de entrenamiento en el uso de herramientas y falta de recursos, o de conocimiento de la existencia de los mismos ${ }^{2,38-41}$, problemas todos ellos que nos resultan familiares y que tendríamos que investigar en nuestro propio medio.

\section{¿Cómo realizar la vigilancia \\ del desarrollo? Herramientas}

Tan solo el $15-20 \%$ de los pediatras estadounidenses utilizan un instrumento normalizado para identificar a los niños con problemas del desarrollo ${ }^{42,43} ; 7$ de cada 10 pediatras norteamericanos reconocen que se fían de su juicio clínico cuando por este método se diagnostican -antes de la escolarización- menos del $30 \%$ de niños con patologías como retraso mental, trastornos de lenguaje o de aprendizaje y otros problemas del desarrollo y menos del $50 \%$ de niños con problemas emocionales o de conducta.

Pero, ¿cómo vigilar y valorar el desa- rrollo infantil? No existe consenso sobre la manera óptima de hacer la supervisión del desarrollo psicomotor aunque la tendencia parece ser la utilización de alguna herramienta. Se recomienda empero utilizar aquellos test validados para la población de referencia o los más cercanos a ella. Así y todo, la supervisión del desarrollo psicomotor basado en escalas de desarrollo o en las preocupaciones que manifiestan los padres tiene una calidad de evidencia científica y un grado de recomendación de tipo II-2 $\mathrm{C}^{44}$.

La Canadian Guide to Clinical Preventive Health Care hace una recomendación en contra de la utilización de una de las herramientas de cribado más extendidas: el test de Denver, y afirma que la evidencia es insuficiente para aconsejar o desaconsejar la utilización de otras herramientas de cribado. Un reciente estudio de la United States Preventive Task Force concluye también que hay evidencia insuficiente para recomendar o no la utilización de instrumentos de cribado en AP para detectar problemas de lenguaje en niños hasta los 5 años de edad ${ }^{46}$. Sin embargo, no existen estudios aleatorizados sobre cribado versus no cribado en niños con las herramientas actuales ${ }^{43}$, por lo que es un tema que continúa abierto a la investigación. 
Hay que distinguir entre lo que son herramientas de detección (o cribado) que se dirigen a detectar niños de riesgo y herramientas de evaluación por las que se identifican los trastornos de desarrollo específicos que afectan a los niños de riesgo identificados por el cribado.

Las herramientas de valoración del desarrollo pueden ser a su vez herramientas generales -que valoran todas las áreas del desarrollo- o específicas. Estas últimas pueden ser, a su vez, específicas para la identificación de un tipo de trastorno del desarrollo -como por ejemplo para la detección de autismo- o específicas de un área del desarrollo -como las utilizadas para la detección o valoración de los problemas de lenguaje-.

Las herramientas de evaluación están basadas en escalas de desarrollo evolutivo, siendo la más conocida la escala de Gesell de 1947, en la que se sustentan casi todas las posteriores (Griffits 1954, Bayley 1969, Uzgiris Hunt 1975, etc.) que consumen mucho tiempo y requieren bastante entrenamiento ${ }^{47}$. Antes de los 2 años de edad los test de desarrollo tienen un fuerte valor predictivo tan solo en casos de niños severa y profundamente retrasados ${ }^{33}$.

De modo que las herramientas de cribado se han desarrollado más reciente- mente para simplificar estos problemas y ahorrar tiempo, y, además, cumplen las normas de estandarización, fiabilidad y validez; a su vez, comparadas con el gold standard de los test de desarroIlo, han demostrado una sensibilidad y especificidad entre el $70-80 \%$, lo que es aceptable dada la complejidad y evolutividad del desarrollo infanti $\left.\right|^{9,35}$.

Sin embargo, es tal la plétora de instrumentos y la dificultad para interpretar los resultados que, a veces, los pediatras siguen adoptando la actitud de guiarse por instinto basándose en su experiencia, o bien utilizar listados con los hitos del desarrollo $u$ otra serie de medios informales ${ }^{2,41,48}$ pese a las recomendaciones actuales.

Además, aunque el pediatra utilice un instrumento de cribado validado, comete frecuentemente alguno de estos errores: utiliza las herramientas selectivamente en aquellos niños en los que el problema de desarrollo es ya evidente y no de forma sistemática en todos los niños que acuden a su consulta, utiliza parcialmente el instrumento comprobando solo alguno de sus ítems, o, en ocasiones, utiliza el test y detecta el problema pero adopta la actitud de esperar y ver sin referir al niño a un servicio de valoración y tratamiento ${ }^{41,49}$. 
Otro argumento que se esgrime en contra de la utilización de los test de cribado es el miedo a los falsos positivos y la consiguiente sobrederivación de niños. Sin embargo, parece que el $70 \%$ de los falsos positivos en dichos test son niños que puntúan por debajo del percentil 25 en una o más variables de los test diagnósticos (inteligencia, lenguaje, conducta adaptativa y rendimiento académico); variables que constituyen los mejores predictores del éxito escolar. Por tanto, estos niños constituyen un grupo de riesgo para los que este cribado supone un beneficio al identificar casos que se pueden beneficiar de tratamientos específicos ${ }^{50}$.

Existen múltiples herramientas de cribado o de diagnóstico para los problemas de desarrollo, bien estandarizados y con aceptables niveles de sensibilidad/especificidad $y$, aunque muchos de ellos han sido traducidos a nuestro idioma (tabla IV) habría que realizar un ímprobo trabajo de validación de los mismos en nuestro medio.

Debemos recordar que se recomienda utilizar preferentemente aquellos instrumentos estandarizados y validados para la población de referencia. En nuestro país parece aconsejable como instrumento de cribado difundir más la utilización de la escala Haizea-Llevant ${ }^{12}$, ta- bla de desarrollo de 0 a 5 años, estandarizada en una muestra representativa de 817 niños del País Vasco y de 1.702 niños de Cataluña. Aunque no existen estudios comparativos entre este y otros instrumentos, ni tampoco sobre la validación de otro tipo de test en población española, la validación objetiva (estadística) supone un esfuerzo tal que se piensa que es más aconsejable empezar a utilizarlos para realizar más bien una validación subjetiva -mediante el uso y comprobación de su rendimiento-.

\section{¿Cómo llevar a la práctica la vigilancia del desarrollo en nuestra consulta?}

En una encuesta nacional más del $40 \%$ de padres estadounidenses de niños menores de 3 años refiere que su hijo nunca recibió una valoración formal ni informal de su desarrollo ${ }^{51}$; aquellos padres que la recibieron manifestaron mayor satisfacción con la atención médica del niño y mayor cumplimentación en los controles de salud.

Además, según algunos estudios, parece que sigue existiendo, como en años precedentes, un desfase importante entre el momento en que los padres refieren preocupaciones sobre el desarrollo de los niños y la edad a la que estos reciben diagnóstico o tratamiento ${ }^{52}$. 
La mayoría de los padres de niños con alteraciones del desarrollo tienen preocupaciones a lo largo del segundo año de vida de este, pero la valoración por un especialista no tiene lugar hasta los 3,5-4 años, comentando las familias que el pediatra quitó importancia a sus preocupaciones inicialmente o les comentó que era demasiado pronto para afirmar si aquello era un problema $a^{53,54}$. En general, los niños con retrasos y/o problemas menos graves $-\mathrm{y}$ que pue-

Tabla IV. Instrumentos disponibles en castellano

\begin{tabular}{|c|c|c|c|c|}
\hline Herramienta & Edad & Tipo batería & $\begin{array}{l}\text { Tiempo } \\
\text { administración }\end{array}$ & $\begin{array}{l}\text { Propiedades } \\
\text { psicométricas }\end{array}$ \\
\hline $\begin{array}{l}\text { Ages and Stages } \\
\text { Questionnaires (ASQ) }\end{array}$ & $\begin{array}{l}\text { 4-60 meses } \\
\text { padres }\end{array}$ & Cuestionario & $10-15$ minutos & $\begin{array}{l}\text { S: } 70-90 \% \\
\text { E: } 76-91 \%\end{array}$ \\
\hline $\begin{array}{l}\text { Batelle Developmental } \\
\text { Inventory Screening Tool } \\
2 .{ }^{\text {nd }} \text { ed. (BDI-ST) }\end{array}$ & $0-95$ meses & $\begin{array}{l}\text { Administración } \\
\text { directa }\end{array}$ & $\begin{array}{l}\text { 10-15 minutos } \\
(<3 \text { años) } \\
\text { 20-30 minutos } \\
\text { (> } 3 \text { años) }\end{array}$ & $\begin{array}{l}\text { S: } 72-93 \% \\
\text { E: } 79-88 \%\end{array}$ \\
\hline $\begin{array}{l}\text { Bayley Infant } \\
\text { Neurodevelopmental } \\
\text { Screen (BINS) }\end{array}$ & 3-24 meses & $\begin{array}{l}\text { Administración } \\
\text { directa }\end{array}$ & 10 minutos & $\begin{array}{l}\text { S: } 75-86 \% \\
\text { E: } 75-86 \%\end{array}$ \\
\hline Brigance Screens II & $0-90$ meses & $\begin{array}{l}\text { Administración } \\
\text { directa }\end{array}$ & 10-15 minutos & $\begin{array}{l}\text { S: } 70-80 \% \\
\text { E: } 70-80 \%\end{array}$ \\
\hline $\begin{array}{l}\text { Child Development } \\
\text { Inventory (CDI) }\end{array}$ & $\begin{array}{l}18 \text { meses a } \\
6 \text { años }\end{array}$ & $\begin{array}{l}\text { Cuestionario } \\
\text { padres }\end{array}$ & $30-50$ minutos & $\begin{array}{l}\text { S: } 80-100 \% \\
\text { E: } 94-96 \%\end{array}$ \\
\hline $\begin{array}{l}\text { Child Development } \\
\text { Review-Parent } \\
\text { Questionnaire (CDR-PQ) }\end{array}$ & $\begin{array}{l}18 \text { meses a } \\
5 \text { años }\end{array}$ & $\begin{array}{l}\text { Cuestionario } \\
\text { padres }+ \\
\text { tabla desarrollo }\end{array}$ & $10-20$ minutos & $\begin{array}{l}\text { S: } 68 \% \\
\text { E: } 88 \%\end{array}$ \\
\hline Denver II & 0-6 años & $\begin{array}{l}\text { Administración } \\
\text { directa }\end{array}$ & $10-20$ minutos & $\begin{array}{l}\text { S: } 56-83 \% \\
\text { E: } 43-80 \%\end{array}$ \\
\hline $\begin{array}{l}\text { Infant Development } \\
\text { Inventory }\end{array}$ & $0-18$ meses & $\begin{array}{l}\text { Cuestionario } \\
\text { padres }\end{array}$ & 5-10 minutos & $\begin{array}{l}\text { S: } 85 \% \\
\text { E: } 77 \%\end{array}$ \\
\hline $\begin{array}{l}\text { Parents'Evaluation } \\
\text { of Developmental } \\
\text { Status (PEDS) }\end{array}$ & 0-8 años & $\begin{array}{l}\text { Cuestionario } \\
\text { padres }\end{array}$ & 2-10 minutos & $\begin{array}{l}\text { S: } 74-79 \% \\
\text { E: } 70-80 \%\end{array}$ \\
\hline $\begin{array}{l}\text { Capute Scales } \\
\text { (CAT / CLAMS) }\end{array}$ & 3-36 meses & $\begin{array}{l}\text { Administración } \\
\text { directa }\end{array}$ & $15-20$ minutos & $\begin{array}{l}\text { S: } 21-67 \% \\
\text { E: } 95-100 \%\end{array}$ \\
\hline $\begin{array}{l}\text { Modified Checklist } \\
\text { For Autism in Toddlers } \\
\text { (M-CHAT) }\end{array}$ & 16-48 meses & $\begin{array}{l}\text { Cuestionario } \\
\text { padres }\end{array}$ & $5-10$ minutos & $\begin{array}{l}\text { S: } 85-87 \% \\
\text { E: } 93-99 \%\end{array}$ \\
\hline $\begin{array}{l}\text { Social Communication } \\
\text { Questionnaire (Autism } \\
\text { Screening Questionnaire } \\
\text { [ASQ]) }\end{array}$ & $\begin{array}{l}4 \text { o más } \\
\text { años }\end{array}$ & $\begin{array}{l}\text { Cuestionario } \\
\text { padres }\end{array}$ & 5-10 minutos & $\begin{array}{l}\text { S: } 85 \% \\
\text { E: } 75 \%\end{array}$ \\
\hline
\end{tabular}

S: sensibilidad; E: especificidad. 
den tener respuestas más significativas al tratamiento- son los menos identificados precozmente ${ }^{27}$.

Conscientes de todos estos problemas las sociedades científicas hacen recomendaciones sobre la mejor forma de realizar la monitorización del desarrollo psicomotor por el pediatra de $\mathrm{AP}^{9,55}$. Actualmente, más que una vigilancia del desarrollo se recomienda el examen sis- temático de todos los niños utilizando una herramienta validada ${ }^{27}$.

La mayoría de los expertos recomienda realizar este cribado a lo largo de los 3 primeros años de vida y otros recomiendan hacer un segundo despistaje más tardío entre los 4 y los 5 años ${ }^{56}$ que serviría para detectar aquellos problemas de desarrollo más leves que pudieran dar falsos negativos en los primeros años.

Figura 4. Algoritmo de vigilancia del desarrollo.

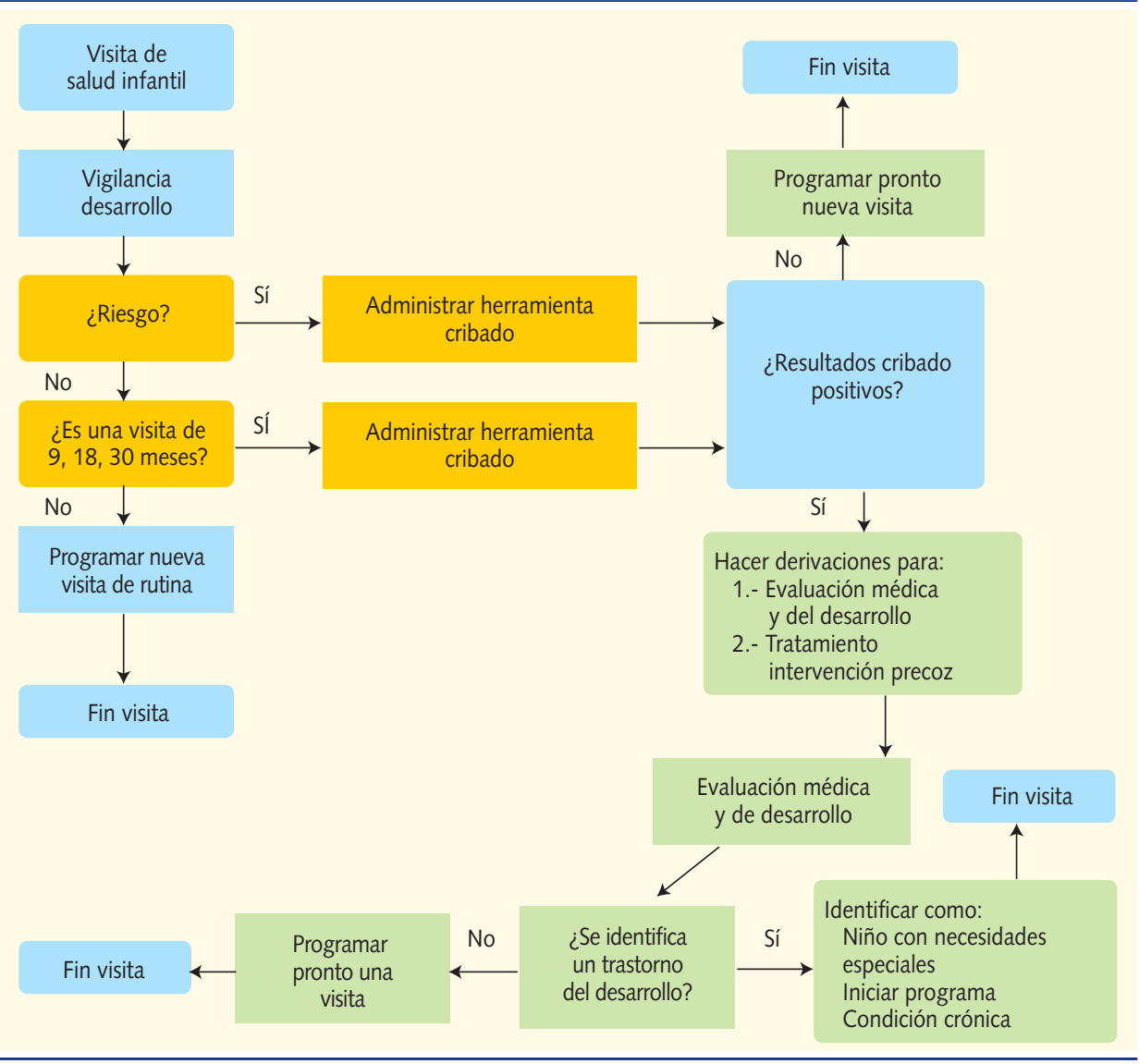


La Academia Americana de Pediatría resume estas recomendaciones en un algoritmo sencillo y práctico como estrategia para ayudar a los pediatras de AP a vigilar el desarrollo de los niños desde el nacimiento hasta los 5 años ${ }^{55}$. La recomendación se resume en hacer un cribado a todos los niños, utilizando una herramienta estandarizada, en tres edades específicas: a los 9, 18 y 24 ó 30 meses, con un cribado adicional para el autismo a los 18 meses (figura 4).

Aunque hay pocos estudios, parece que la implementación de esta práctica aumenta el porcentaje de cribado del desarrollo del $15 \%$ a más del $70 \%$ en las visitas de salud infantil ${ }^{57}$. Y este aumento se traduce en un incremento de las derivaciones al programa de Atención Temprana del 2,6\% al 7-8\% de niños después de la implementación ${ }^{58}$.

\section{Conclusiones}

Vemos en nuestra muestra que la prevalencia de retrasos/trastornos del desarrollo o situación de riesgo para el desarrollo es relevante: el $8,68 \%$ de la población general de 0-6 años atendida en nuestras consultas de Pediatría de AP. El $51,21 \%$ de ellos evolucionan hacia algún tipo de patología, lo que supondría el $4,4 \%$ de la población general entre 0 y 6 años.
El $73 \%$ de estos niños presentaban uno o más factores de riesgo biológico y/o social, pero el $27 \%$ de ellos no los tenían; en ambos grupos hubo niños que evolucionaron hacia algún tipo de patología, por lo que la vigilancia del desarrollo por parte del pediatra debe ser universal independientemente de la presencia o no de dichos factores de riesgo.

Atendiendo a la evolución de los niños que no presentaron inicialmente ni factores de riesgo ni problemas de desarrollo se produce un incremento de patología, de modo que el 11,2\% de la población general de 0-6 años presenta a lo largo del tiempo algún tipo de problema del desarrollo ya sea retraso mental, TDAH, trastornos de aprendizaje, etc.

De los niños que no accedieron a tratamiento de Atención Temprana, el $36,3 \%$ evolucionó hacia algún tipo de patología. De ahí, la conveniencia de tratar no solo niños con discapacidades establecidas sino también aquellos en situación de riesgo de padecerlas como se intenta llevar a cabo actualmente en la mayoría de programas de Atención Temprana.

Actualmente existen pocos estudios sobre detección de problemas del desarrollo en nuestro medio de Pediatría de 
$A P$, por lo que es necesario seguir investigando en este sentido.

Es necesario identificar las barreras por las que existe tanta diferencia entre la prevalencia de niños con problemas del desarrollo y el retraso en su identificación.

También hay que insistir en la elaboración y utilización universal y sistemática de herramientas de cribado del desarrollo infantil de suficiente calidad, que puedan ser incorporadas fácilmente a las historias informáticas de nuestros pacientes.

Es necesario, además, entrenar a los residentes de Pediatría en la utilización de estas herramientas y estrategias que tendrán que implantar en su futuro profesional fuera del medio hospitalario.
Es preciso documentar la efectividad, a todos los niveles, de la implementación de estas u otras estrategias en nuestro medio de $\mathrm{AP}^{24}$.

$Y$, finalmente, es imperante adoptar políticas universales en este sentido, de modo que eliminemos las diferencias entre comunidades y logremos que todos los niños puedan acceder a los programas de intervención precozmente.

\section{Agradecimientos}

A todos los orientadores escolares que han facilitado el seguimiento de estos niños y en especial a los profesionales de nuestro centro educativo de referencia CP Mendillorri.

\section{Bibliografía}

1. Dworking PH. Detection of behavioral, developmental and psychosocial problems in pediatric primary care practice. Curr Opin Pediatr. 1993;5:531-6.

2. Sices L, Feudtner C, Mc Lauhlin J, Drotar D, Williams $M$. How do primary care physicians identify young children with developmental delays? A national survey. J Dev Behav Pediatr. 2003;24:409-17.

3. Contreras MM. Clasificaciones diagnósticas de los trastornos del desarrollo. En: Lejarraga $\mathrm{H}$. Desarrollo del niño en contexto. Buenos Aires: Ed. Paidós SAICF; 2004. p. 143-92.
4. Shevell $M$, Ashwall S, Donley D, Flint J, Gingold $M$, Hirtz $D$, et al. Practice parameter: evaluation of the child with global developmental delay. Report of the quality standards subcommittee of the American Academy of Neurology and the practice committee of the Child Neurology Society. Neurology. 2003;60:36780.

5. Narbona J, Schlumberger E. Retraso psicomotor. Protocolos de Neurología de la Asociación Española de Pediatría y Sociedad Española de Neurología 2008 [consultado el 15/08/2008]. Disponible en www.aeped.es/protocolos/neuro logia/index.htm

6. Accardo PJ, Whitman BY. Dictionary of De- 
velopmental Disabilities terminology. 2. ${ }^{a}$ ed. Londres: Paul Brookes; 2001.

7. Czornyj LA. Retraso madurativo. Aspectos neurológicos. En: Lejarraga $\mathrm{H}$. Desarrollo del niño en contexto. Buenos Aires: Ed. Paidós SAICF; 2004. p. 193-215.

8. Rydz D, Shevell M, Majnemer A, Oskoui M. Topical review: developmental screening. J Child Neurol. 2005;20:4-21.

9. American Academy of Pediatrics. Committee on Children with Disabilities. Developmental surveillance and screening of infants and young children. Pediatrics. 2001;108:192-6.

10. King-Thomas L, Hacker BJ. A therapist's guide to pediatric assessment. Dev Behav Pediatr. 1991;12:42-50.

11. Instituto Nacional de Estadística (INE). Encuesta 1999 sobre Discapacidad, Deficiencia y Estado de Salud. Madrid: INE; 2000 [consultado el 12/01/2009]. Disponible en www.ine.es/ prodyser/pubweb/discapa/disctodo.pdf

12. Fuentes J, Fernández-Matamoros I, Rueda P. Escala Haizea. Vitoria: Servicio Central de Publicaciones del Gobierno Vasco; 1991.

13. Bayley N. BSID. Escalas Bayley de desarroIlo infantil. Madrid: TEA Ediciones S.A. The Psychological Corporation; 1977.

14. Jonson-Martin NM, Jens KG, Attermeier SM, Hacker BJ. Currículo Carolina. Evaluación y ejercicios para bebés y niños pequeños con necesidades especiales. Madrid: TEA Ediciones S.A.; 1997.

15. Guralnick MJ. The effectiveness of early intervention. Baltimore, Maryland: Paul Brookes Publishing Co; 2000.

16. Federación Estatal de Asociaciones de Profesionales de Atención Temprana. Libro Blanco de la Atención Temprana. Documento 55/2005. Madrid: Ministerio de Trabajo y Asuntos Sociales. Real Patronato de Prevención y de Atención a personas con minusvalías; 2005.

17. Barnett SW. Long term effects of early childhood programs on cognitive and school outcomes. Future Child. 1995;5:25-50.

18. Majnemer A. Benefits of early intervention for children with developmental disabilities. Semin Pediatr Neurol. 1998;5:62-9.

19. Reynolds AJ, Temple JA, Robertson DL, Mann EA. Long term effects of an early childhood intervention on educational achievement and juvenile arrest. JAMA. 2001;285:2339-46.

20. Campbell FA, Pungello EP, Miller-Johnson S, Burchinal M, Ramey C. The development of cognitive and academic abilities: growth curves from an early childhood educational experiment. Dev Psychol. 2001;37:231-42.

21. Ramey C, Campbell F, Burchinal M, Skinner M, Gardner D, Ramey S. Persistent effects of early intervention on high-risk children and their mothers. Applied Dev Science. 2000;4:2-14.

22. Mc Cormick M, Brooks-Gunn J, Buka S, Goldman J, Yu J. Early intervention in Low Birth Weight Premature Infants: results at 18 years of age for the Infant Health and Development Program. Pediatrics. 2006;117:771-80.

23. Federación Estatal de Asociaciones de Profesionales de la Atención Temprana. Organización Diagnóstica para la Atención Temprana (ODAT). Madrid: Real Patronato sobre Discapacidad; 2004.

24. Zamarriego J, Arizcun J. Introducción al Simposium Internacional sobre experiencias y resultados en programas de prevención de la subnormalidad. Madrid: Libro de Actas; 1981. p. 20-41.

25. Ramos Sánchez I, Márquez Luque A. Recién nacidos de riesgo neurológico. Vox Paediatr. 2000;8:5-10.

26. American Academy of Pediatrics. Council on children with disabilities. Role of the medical 
home in family-centered early intervention services. Pediatrics. 2007;120:1153-8.

27. Sices L. Developmental screening in primary care: the effectiveness of current practice and recommendations for improvement. Commonwealth Fund Pub. 2007 [consultado el 12/01/2009]. Disponible en www.commonwe althfund.org/usr_doc/1082_Sices_developmen tal_screening_primary_care.pdf?section $=4039$.

28. Documento marco sobre la Atención Temprana en Navarra. Borrador 5. Navarra: INBS (Instituto Navarro de Bienestar Social); 2004.

29. Álvarez Urricelqui MJ. Atención Temprana en Navarra. Minusval. 2004;145:16-7.

30. Confederación Española de Organizaciones a favor de las Personas con Discapacidad Intelectual (FEAPS) en colaboración con la Asociación Española de Pediatría (AEP) y Obra Social Caja Madrid. Campaña de información y sensibilización sobre Atención Temprana hacia pediatras (octubre 2004) [consultado el 12/01 /2009]. Disponible en www.anisalud. com/fiche ros/carpeta.doc y www.astait.org/feaps-aep. htm

31. Bear L. Early identification of infants at risk for developmental disabilities. Pediatr Clin North Am. 2004;51:685-701.

32. Council of children with disabilities; section on developmental behavioral pediatrics; Bright Futures Steering Committee; medical home initiatives for children with special needs project advisory committee. Identifying infants and young children with developmental disorders in the medical home. An algorithm for developmental surveillance and screening. American Academy of Pediatrics. Policy Statement. Pediatrics. 2006;118:405-20.

33. Aicardi J. Diseases of the nervous system in childhood. Clinics in developmental medicine N. ${ }^{\circ}$ 115/118. London: Mac Keith Press; 1992.
34. Illingworth RS. El niño normal. México DF: Manual Moderno S.A.;1993.

35. Glascoe FP, Macias MM. How you can implement the AAP's new policy on developmental and behavioral screening. Contemporary Pediatrics. 2003;20:85-102.

36. Palfrey JS, Singer JD, Walker DK, Butler JA. Early identification of children's special needs: a study in five metropolitan communities. J Pediatr. 1987;111:651-9.

37. Academy of Pediatrics. Division of health policy research. Periodic survey identification of children < 36 months at risk for developmental problems and referral to early identification programs 2003 [consultado el 12/01/2009]. Disponible en www.aap.org/research/periodicsur vey/ps53exs.htm

38. Sices L, Feudtner C, Mc Laughlin J. How do Primary Care Physicians manage children with possible developmental delays? A national survey with an experimental design. Pediatrics. 2004;113:274-82

39. American Academy of Pediatrics, Committee on Practice and Ambulatory Medicine. Recommendations for preventive pediatric health care (RE9939). Pediatrics. 2000;105:645-6.

40. American Academy of Pediatrics. Guidelines for health supervision III, 3.rd ed. ELK Grove Village: American Academy of Pediatrics; 2002.

41. Sand N, Silverstein M, Glascoe FP. Pediatricians' reported practices regarding developmental screening: do guidelines work? Do they help? Pediatrics. 2005;116:174-9.

42. Smith RD. The use of developmental screening tests by primary care pediatricians. J Pediatr. 1978;93:524-7.

43. Hamilton S. Screening for developmental delay: reliable, easy-to-use tools. J Fam Practice. 2006;55:415-22.

44. Soriano FJ, Delgado JJ, Galbe J, Pericas J, 
Merino M, Sánchez FJ, y cols. Manual de actividades preventivas en la infancia y adolescencia. PrevInfad/PAPPS. Grupo de trabajo de prevención en la infancia y adolescencia de la Asociación Española de Pediatría de Atención Primaria. Madrid: Exlibris Ediciones; 2004.

45. Canadian Task Force (CTF).The Canadian Guide to clinical preventive health care. CTFPHC systematic reviews and recommendations. Pediatric Preventive Care: Preschool screening for Developmental Problems; 1994 [consultado el 12/01/2009]. Disponible en www.ctfphc.org/ guide.htm [actualizado en 2003].

46. US Preventive Services Task Force. Screening for speech and language delay in preschool children [consultado el 12/01/2009]. Disponible en www.ahrq.gov/clinic/USpstf/uspschdv.htm [actualizado en 2006].

47. Rosenbaum P. Screening test and standardized assessments used to identify and characterize developmental delays. Sem Pediatr Neurology. 1998;5:27-32.

48. Glascoe F. Detecting and addressing developmental and behavioural problems in primary care. Pediatr Nurs. 2000;26:251-66.

49. Glascoe F, Shapiro H. Introduction to developmental and behavioral screening. Developmental Vehavioral Pediatrics Online [Internet] [consultado el 12/01/2009]. Disponible en www.dbpeds.org/articles/detail.cfm?id=5 [actualizado en 05/02/2007].

50. Glascoe F. Are overreferrals on developmental screening test really a problem? Arch Pediatr Adolesc Med. 2001;155:54-9.

51. Halfon N, Regalado M, Sareen H. Assesing development in the pediatric office. Pediatrics.
2004;113 Suppl 6:1926-33.

52. Lock T, Shapiro B, Ross A. Age of presentation in developmental disability. J Dev Behav Pediatr. 1986;7:340-45.

53. Shevell MI, Majnemer A, Rosenbaum. Profile of referrals for early childhood developmental delay to ambulatory subspecialty clinics. J Child Neurol. 2001;16:645-50.

54. Bailey D, Skinner D, Hatton D. Family experiences and factors associated with the diagnosis of fragile $X$ syndrome. J Dev Behav Pediatr. 2000;21:315-21.

55. Council of children with disabilities; section on developmental behavioral pediatrics; Bright Futures Steering Committee; medical home initiatives for children with special needs project advisory committee. Identifying infants and young children with developmental disorders in the medical home. An algorithm for developmental surveillance and screening. American Academy of Pediatrics. Policy Statement. Pediatrics. 2006;118:405-20.

56. Sociedad Argentina de Pediatría. Guías de seguimiento de niños y adolescentes. 2. ${ }^{a}$ ed. Buenos Aires: Sociedad Argentina de Pediatría; 2002.

57. Earls MF, Hay SS. Setting the stage for success: implementation of developmental and behavioral screening and surveillance in primary care practice - the North Carolina assuring better child health and development (ABCD) project. Pediatrics. 2006;118:183-8.

58. Pinto-Martín JA, Dunkle M, Earls M. Developmental stages of developmental screening: steps to implementation of a successful program. Am J Public Health. 2005;95:1928-32. 